\title{
Article
}

Doi 10.5943/sif/ 2/1/11

Copyright $\odot$ Mushroom Research Foundation

\section{Diversity of macrofungi and its distribution pattern of Gorakhpur District, Uttar Pradesh, India}

\author{
Vishwakarma $P$, Singh $P$ and Tripathi NN
}

Bacteriology and Natural Pesticide Laboratory, Department of Botany, DDU Gorakhpur University, Gorakhpur, 273009, UP, India

Vishwakarma P, Singh P, Tripathi NN 2017 - Diversity of macrofungi and its distribution pattern of Gorakhpur District, Uttar Pradesh, India. Studies in Fungi 2(1), 92-105, Doi 10.5943/sif/2/1/11

\begin{abstract}
The present study deals with the status of macrofungal diversity in Gorakhpur district and its distribution pattern. The macrofungal survey was undertaken during 2011-2014 in different localities of Gorakhpur district. A total of 114 species of macrofungi belonging to 58 genera and 33 families were collected and identified in to 31 edible species, 10 excellent edible species, 68 inedible species and 5 poisonous species. Agaricaceae family was found to be the dominant representing 18 species. Distribution of macrofungal species in different localities of Gorakhpur district was also evaluated on the basis of Shannon diversity index, Simpson diversity index and evenness. Highest Shannon diversity index, Simpson diversity index and evenness were found to be 3.61, 0.97 and 0.90 respectively in Sahjanwan tehsil. The results indicate a very high species richness of the study site.
\end{abstract}

Key words - Agaricales - Basidiomycota - Diversity index - Edible macrofungi

\section{Introduction}

Macrofungi are cosmopolitan, heterotrophic organisms that are quite specific in their nutritional and ecological requirements. Macrofungi occupy important place in the biodiversity of India. Macrofungi (also called mushrooms) are represented by 41,000 species across the globe out of which only $\sim 2 \%$ have been reported from India, despite the fact that one-third of the total global fungal diversity exists in the tropical Indian region (Priyamvada et al. 2017). Many Asian countries use traditionally wild edible mushrooms as delicious and nutritional food and medicine. Wild edible macrofungi are appreciated not only for texture and flavour but also for their chemical and nutritional characteristics (Tapwal et al. 2013).

From time to time different workers had studied macrofungal diversity from Northern part of India which includes North Western region, Eastern Himalaya proper and North Eastern hilly areas. Lots of macrofungi were collected from Himachal Pradesh by Sohi et al. (1964, 1965). Ghosh \& Pathak (1965) collected 3 species of Macrolepiota from Lucknow (U.P.), Ghosh et al. (1967) also described about some edible macrofungi of Lucknow. Chandrawati et al. (2014) collected 29 species of macrofungal belonging to 12 families from Gorakhpur while Vishwakarma et al. (2014) reported 12 taxa of macrofungi belonging to 8 families from here. Vishwakarma et al. (2017a) had provided a complete checklist of macrofungi of Gorakhpur district. Some works on nutritional property of macrofungi of this area were also carried out by Vishwakarma et al. 2016, 2017b. 
North Eastern part of Uttar Pradesh is endowed with a rich biodiversity of flora and fauna. It is situated in Terai region of Himalaya and hence provides suitable climatic and environmental condition for successful establishment of all types of flora especially the macrofungi. So the main aim of the present study was to explore and identify the macrofungal diversity of Gorakhpur district.

\section{Materials \& Methods}

\section{Study area}

Gorakhpur is situated in North Eastern part of Uttar Pradesh. Gorakhpur district is a one of the major and largest district of Uttar Pradesh. Gorakhpur is the headquarter of Gorakhpur division and district both. Gorakhpur district is divided into seven Tehsils (Revenue Sub-division) presided over by a Sub-Divisional Magistrate. It occupies an area of about 7,483.8 $\mathrm{Km}^{2}$ with latitude and longitude of $26^{\circ} 43^{\prime}$ to $26^{\circ} 50^{\prime} \mathrm{N}$ and $83^{\circ} 20^{\prime}$ to $83^{\circ} 27^{\prime} \mathrm{E}$ respectively. The annual average temperature is $25-30^{\circ} \mathrm{C}$. Gorakhpur region receives total annual average rainfall of about $1814 \mathrm{~mm}$, about $87 \%$ annual rainfall is received during warm rainy season and rest $13 \%$ is distributed in the form of occasional shower from November to May. Relative humidity ranges between 74-87\%. The soil of this area is genetic alluvial brought down by river Rapti, Rohini, Ghaghara and Gandak from the Himalayas. It is situated on the basin of river Rapti and Rohini hence its geographical shape is a bowl type. Gorakhpur district has good vegetation cover and also have dense forest which is close to the foothills of Himalayas. All the areas of Gorakhpur district are rich in species composition of higher plants. North Eastern part of Uttar Pradesh with its varied topography, diverse vegetation and climatologically fluctuations can be successfully explored for the growth of macrofungi in wild habitat. In spite of the fact this region is rich in resources of edible macrofungi, no planned effort has been made so far to collect and conserve them. There is vast scope of edible macrofungi which grow wild in forests and grasslands of this region. The moderate rainfall in this part supports a rich flora. Collection of samples was done at tehsil level (which includes blocks and then villages). Different tehsil visited during the survey were Sahjanwan, Sadar, Khajni, Bansgaon, Campierganj and Gola.

\section{Collection, identification and processing of macrofungi}

Regular field trips were carried out in different places of Gorakhpur villages and forests, usually 4-5 times per months. Fruiting body/carpophores with stipe were picked up from substratum on which it was growing with the help of scrapper or knife or forceps and were wrapped individually in wax paper. Samples were photographed with proper scale in its natural habitats for its appropriate identification by using digital camera (Panasonic, DMC-F2). Place and date of collection, habit and habitat were noted down in the field diary at the time of collection and particular collection numbers was given to each specimen.

Samplings were done using quadrate method each measuring $20 \times 20 \mathrm{~m}$. Total of 28 sampling plots in the above 6 sampling sites were studied. Macrofungal specimens were preserved wet as well as dry following Ainsworth (1971). Fruiting bodies of macrofungi were preserved in solution of alcohol $(15 \mathrm{ml})$, formalin $(25 \mathrm{ml})$ and distilled water $(100 \mathrm{ml})$. Dry preservation of macrofungi were done by hot air oven at $40-50^{\circ} \mathrm{C}$ and stored in air tight zip lock polyethylene bags with naphthalene balls for further microscopic studies. The spore prints were taken according to the guideline given by Kuo (2001). Information regarding edibility and other uses of macrofungi species were collected by villagers, local inhabitants of the area and finally by consulting literature. Mycorrhizal associations were ascertained in species found just near the tree base by tracing out the root connection to the fructification.

Specimens were identified using the relevant literature (Alexopolous et al. 1996, Ellis \& Ellis 1990, Jordan 1995, Moser 1983, Phillips 1981) and confirmed by mycokeys (www.mushroomexpert.com and www.mycokeys.com). 


\section{Data analysis} (2012).

Diversity index of macrofungi was calculated as followed by Pushpa \& Purushothama

Shannon diversity index for macrofungi was calculated by using following formula:

$$
H=\Sigma\left(\frac{n}{N}\right) \ln \left(\frac{n}{N}\right)
$$

$\mathrm{H}$ is the diversity index; $\mathrm{N}$ is the total number of individuals of all the species and $\mathrm{n}$ is the total number of individuals of particular species.

Simpson Index of Diversity=1-D

$$
D=\Sigma n(n-1) / N(N-1)
$$

$\mathrm{D}$ is the Simpson's index, $\mathrm{N}$ is the total number of individuals of all species and $\mathrm{n}$ is the total number of organism of a particular species

With the help of the values of diversity index, the evenness of the mushrooms was also calculated as

$$
e=H / \ln S
$$

Where e is evenness, $\mathrm{H}$ is Shannon diversity index and $\mathrm{S}$ is the number of species

\section{Results \& Discussion}

A total of 114 species of macrofungi belonging to 58 genera of 33 families were identified during the survey. Table 1 represents the list of collected species during the survey period and is arranged according to their representative families. Agaricaceae family (18 species) was found to be the most dominant while Clavariaceae, Geastraceae, Helotiaceae, Hygrophoraceae, Meripiliaceae, Meruliaceae, Mycenaceae, Russulaceae, Schizophyllaceae, Sparassidiaceae, Steraceae and Tuberaceae contains only one species each (Fig. 1).

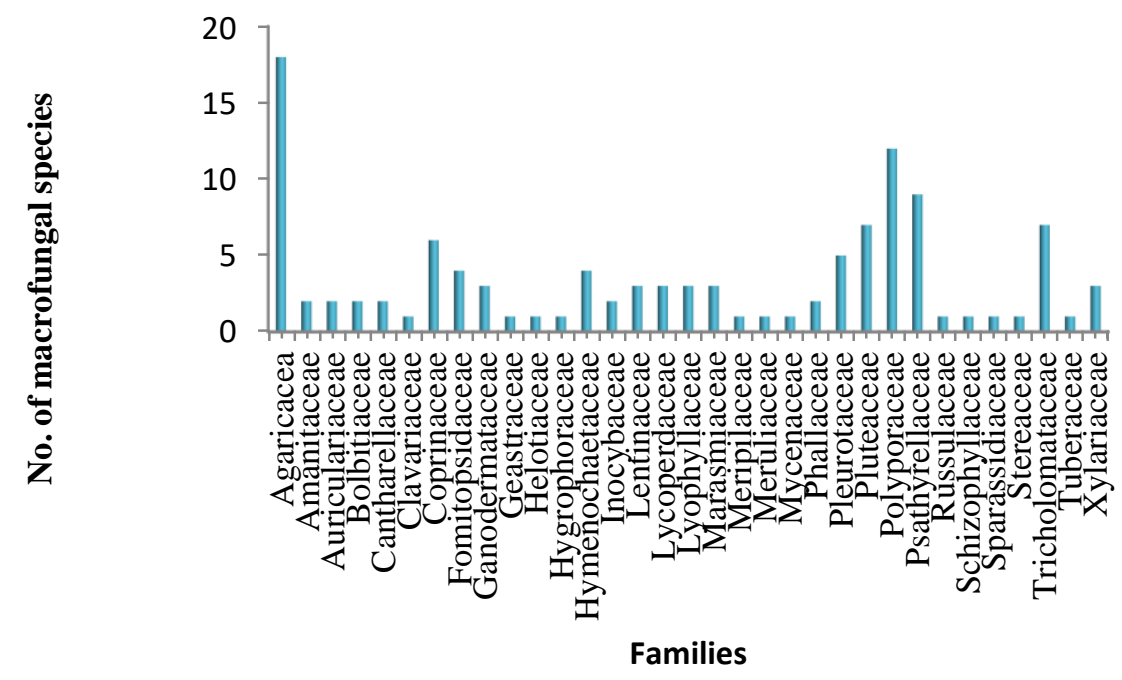

Fig. 1 - Family wise distribution of macrofungi in Gorakhpur district

The diverse climatic conditions of Gorakhpur make this area a natural habitat for a large number of macrofungi. Diversity of macrofungi varies greatly because of different ecological habitats viz., decaying wood logs, humid soil, sandy soil, humus, leaf litters etc. Mushrooms are seasonal fungi, which occupy diverse niches in nature in the forest ecosystem (Pushpa \& Purushothama 2012). In present investigation (Fig. 2) the ecological preference of the species revealed that maximum number of (93) species were saprobic, 12 species were parasitic on higher trees, 6 coprophilous and 3 were symbiotic. The abundance of macrofungi on different substrate greatly depends up on the organic and nitrogenous content of the soil and also on the other nutrients factors which plays key role in the growth of fungi (Kumar et al. 2013). 


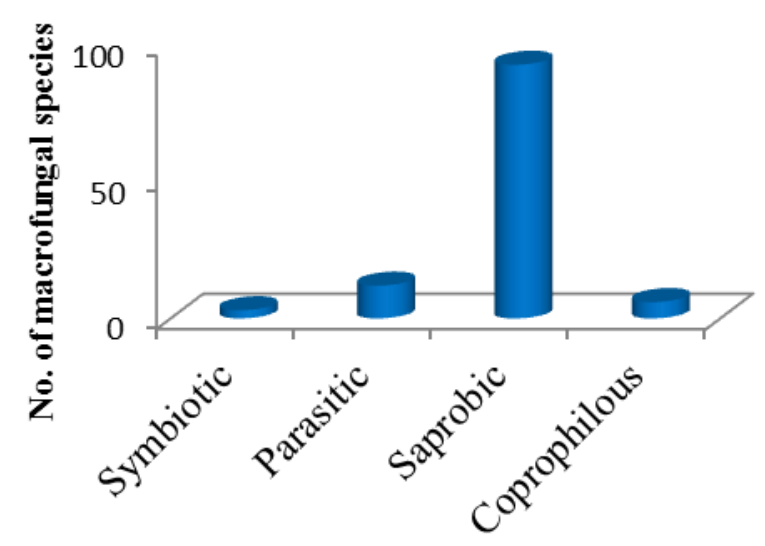

Habitat

Fig. 2 - Distribution of different macrofungi groups

The survey related to the edibility status of macrofungi was also undertaken and the result revealed that out of 114 macrofungal species collected 31 macrofungal species were found to be edible, 10 excellent edible, 68 inedible while 5 poisonous (Fig. 3). Species such as Agaricus, Calocybe, Pleurotus, Termitomyces, Tuber and Volvariella are considered highly delicious. The usage of these macrofungi both commercially and domestically may be due to their appealing taste, frequent occurrence and the fact that they are easily identified by the local peoples as safe for consumption. Macrofungi contains high nutritive value. It contains low fat, rich in fibre, protein, minerals $(\mathrm{Ca}, \mathrm{P}, \mathrm{K})$ and vitamins. It has high nutraceutical value which has created an interest of local peoples towards its use (Abolfazi \& Janardhana 2012).

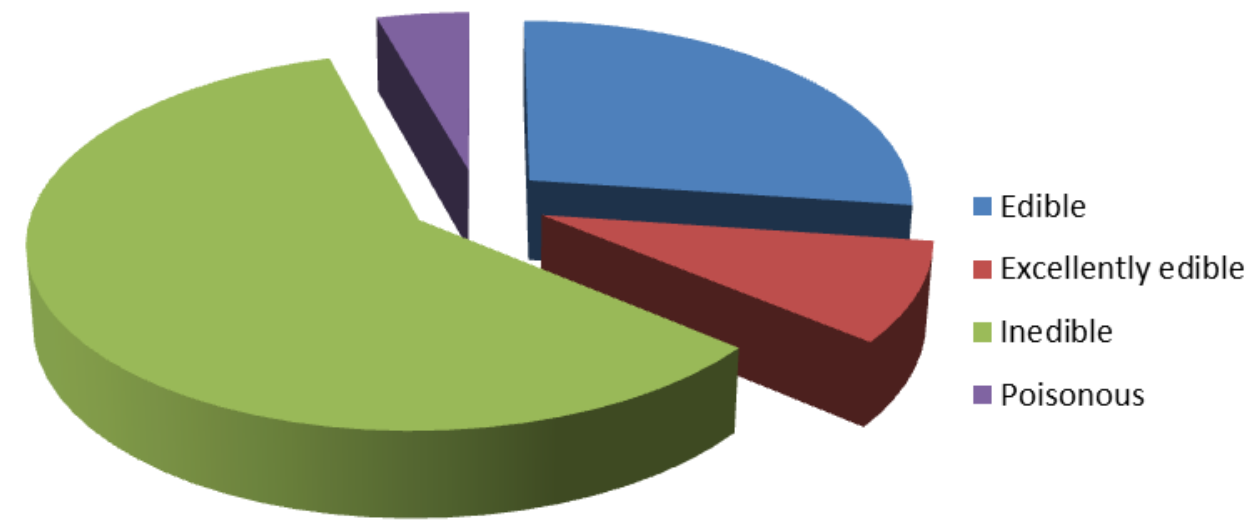

Fig. 3 - Edibility status of collected macrofungi in per cent

Diversity of an area is greatly affected by the environmental condition experience by that particular habitat and this impart variation in occurrence of macrofungi in different seasons. It is clear from the fig 4 that during four year of survey greater number of species were recorded during the rainy season. Months of July followed by August and September each year (2011-2014) contains highest number of macrofungi, while there was complete absence of macrofungal species 
in the months of January and November of all year (Fig. 5). In months of July, August and September the environmental conditions are very favorable for the growth of various macrofungi. Rainy season supports more macrofungal growth. Some woody macrofungi are present throughout the year while some occurs only at specific time of the year.

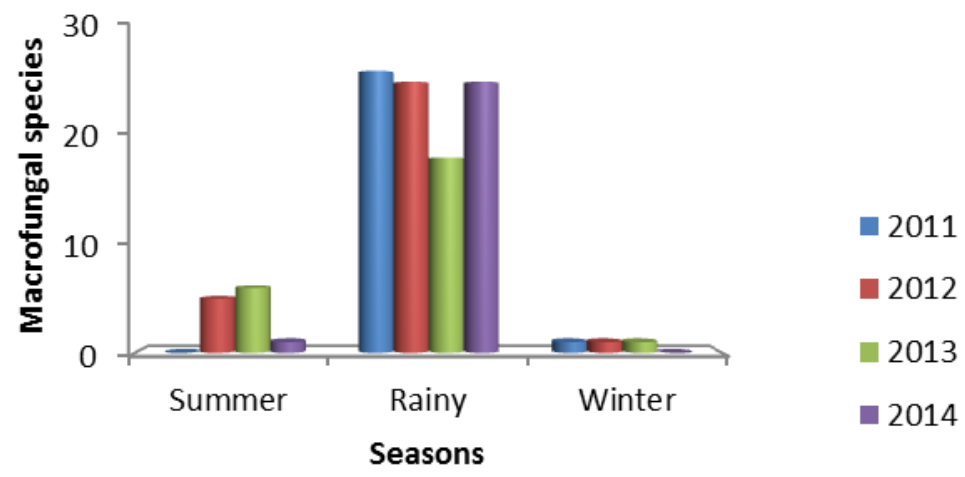

Fig. 4 - Distribution of macrofungal species in different seasons (2011-2014)

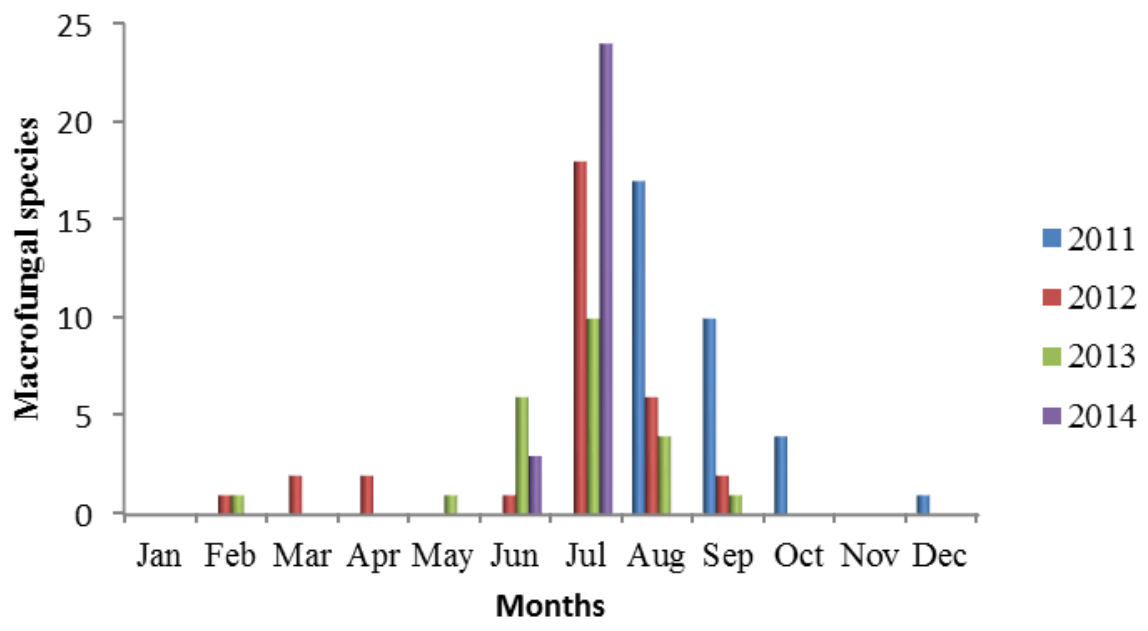

Fig. 5 - Distribution of macrofungal species in different months (2011-2014)

The diversity index is a mathematical representation of species diversity in a community. Diversity indices provide important information about rarity and commonness of species in a community. It takes in to account the number of species present (species richness), as well as the abundance of each species (evenness). Simpson's diversity index (1-D) is a simple method to measure species diversity in a community. The value of $\mathrm{D}$ ranges between 0 and 1 . Greater the value of $\mathrm{D}$ greater is the diversity. Shannon diversity index is commonly used to characterize species diversity in a community. Macrofungi were collected from six different sites of Gorakhpur district to evaluate the macrofungi richness. Species diversity and species richness of macrofungi are shown in table 2. The area wise sampling for macrofungi shows that Sahjanwan tehsil contains highest number of species (53), followed by Sadar tehsil which contains 34 species, Khajni tehsil 
Table 1 Diversity of macrofungi in Gorakhpur District

\begin{tabular}{|c|c|c|c|c|c|}
\hline Voucher no. & Family & Macrofungi & $\begin{array}{c}\text { Date of } \\
\text { collection }\end{array}$ & Ecological habitat & Property \\
\hline DDUNPL206 & Agaricaceae & Agaricus arvensis Schaeffe. & $23 / 9 / 11$ & Saprobic, on grassy area, in group & Excellent edible \\
\hline DDUNPL207 & Agaricaceae & A. bernardii Quèl. & $11 / 7 / 12$ & Saprobic, on grassy area, in group & Excellent edible \\
\hline DDUNPL208 & Agaricaceae & A. bitorquis (Quèl) Sacc. & $5 / 7 / 14$ & Saprobic, on manure in group & Edible \\
\hline DDUNPL209 & Agaricaceae & A. impudicus (Rea) Pilát & $11 / 7 / 12$ & Saprobic, on humus in group & Edible \\
\hline DDUNPL210 & Agaricaceae & A. langei (F.H. Møller) & $5 / 7 / 14$ & $\begin{array}{l}\text { Saprobic, under mixed wood on soil } \\
\text { in group }\end{array}$ & Edible \\
\hline DDUNPL211 & Agaricaceae & A. silvaticus Schaeff. ex.Secr. & $20 / 7 / 12$ & Saprobic, on soil, solitary to in group & Edible \\
\hline DDUNPL212 & Agaricaceae & A. silvicola (Vittad.) Peck & $11 / 8 / 11$ & $\begin{array}{l}\text { Saprobic on decaying litters, solitary, } \\
\text { scattered }\end{array}$ & Edible \\
\hline DDUNPL213 & Agaricaceae & $\begin{array}{l}\text { Chlorophyllum molybdites (G. } \\
\text { Mey.) Massee }\end{array}$ & $3 / 7 / 13$ & $\begin{array}{l}\text { Saprobic, growing gregariously in } \\
\text { lawn and garden }\end{array}$ & Inedible \\
\hline DDUNPL214 & Agaricaceae & C. rhacodes (Vittad.) Vellinga & $9 / 7 / 13$ & $\begin{array}{l}\text { Saprobic, growing in group in } \\
\text { gardens }\end{array}$ & Edible, choice \\
\hline DDUNPL215 & Agaricaceae & Lepiota aspera (Pers.) Quell. & $1 / 10 / 11$ & Saprobic, on leaf litters in group & Edible \\
\hline DDUNPL216 & Agaricaceae & L. atrodisca Zeller & $4 / 7 / 14$ & Saprobic, on leaf litters in group & Inedible \\
\hline DDUNPL217 & Agaricaceae & L. castaneidisca Murrill & $23 / 9 / 11$ & $\begin{array}{l}\text { Saprobic, under hardwood trees } \\
\text { solitary to scattered }\end{array}$ & Inedible \\
\hline DDUNPL218 & Agaricaceae & L. ignivolvata Bousset \& Joss. & $23 / 08 / 11$ & Saprobic on soil & Inedible \\
\hline DDUNPL219 & Agaricaceae & $\begin{array}{l}\text { Leucoagaricus americanus } \\
\text { (Peck) Vellinga }\end{array}$ & $5 / 7 / 13$ & Saprobic on humus, in group & Edible \\
\hline DDUNPL220 & Agaricaceae & L. rubrotinctus (Peck) Singer & 29/9/11 & $\begin{array}{l}\text { Saprobic in group near Bambusa } \\
\text { arundinacea culm }\end{array}$ & Inedible \\
\hline DDUNPL221 & Agaricaceae & $\begin{array}{l}\text { Leucocoprinus brebissonii } \\
\text { (Godey) Locq. }\end{array}$ & $16 / 7 / 12$ & Saprobic on litter, solitary, scattered & Inedible \\
\hline DDUNPL222 & Agaricaceae & L. cepestipes (Sowerby) Pat. & $24 / 2 / 13$ & Saprobic on humus rich soil, in group & Inedible \\
\hline DDUNPL223 & Agaricaceae & $\begin{array}{l}\text { Macrolepiota procera (Scop.) } \\
\text { Singer }\end{array}$ & $23 / 7 / 12$ & Saprobic on decaying litter in group & Edible \\
\hline DDUNPL204 & Amanitaceae & $\begin{array}{l}\text { Amanita cokeri E.-J. Gilbert \& } \\
\text { Kühner ex E.-J. Gilbert }\end{array}$ & 20/7/12 & $\begin{array}{l}\text { Symbiotic with Tectona grandis, } \\
\text { solitary, scattered }\end{array}$ & Inedible \\
\hline DDUNPL205 & Amanitaceae & A. virosa Fr. & $18 / 8 / 11$ & Solitary on humus rich soil & $\begin{array}{l}\text { Highly } \\
\text { Poisonous }\end{array}$ \\
\hline
\end{tabular}


Table 1 (continued)

\begin{tabular}{|c|c|c|c|c|c|}
\hline Voucher no. & Family & Macrofungi & $\begin{array}{c}\text { Date of } \\
\text { collection }\end{array}$ & Ecological habitat & Property \\
\hline DDUNPL163 & Auriculariaceae & $\begin{array}{l}\text { Auricularia auricula-judae } \\
\text { (Bull.) Quél }\end{array}$ & $4 / 7 / 14$ & $\begin{array}{l}\text { Parasitic on healthy tree (Tecoma } \\
\text { capensis), in group }\end{array}$ & Edible \\
\hline DDUNPL164 & Auriculariaceae & A. mesenterica (Dicks.) Pers. & $14 / 7 / 13$ & $\begin{array}{l}\text { Saprobic, in group on decaying wood } \\
\log \end{array}$ & Inedible \\
\hline DDUNPL255 & Bolbitiaceae & $\begin{array}{l}\text { Bolbitius coprophilus (Peck) } \\
\text { Hongo }\end{array}$ & $13 / 7 / 12$ & Coprophilous on compost, in group & Inedible \\
\hline DDUNPL256 & Bolbitiaceae & B. vitellinus (Pers.) Fr. & $8 / 7 / 12$ & $\begin{array}{l}\text { Coprophilous on goat dung, growing } \\
\text { alone scattered }\end{array}$ & Poisonous \\
\hline DDUNPL167 & Cantharellacea & Cantharellus minor Peck & $19 / 8 / 11$ & $\begin{array}{l}\text { Saprobic, growing solitary or in } \\
\text { groups under Bambusa arundinacea }\end{array}$ & Inedible \\
\hline DDUNPL168 & Cantharellacea & C. subalbidus Smith \& Morse & $31 / 8 / 12$ & Saprobic on decaying litter, in group & Edible \\
\hline DDUNPL169 & Clavariaceae & $\begin{array}{l}\text { Clavulinopsis laeticolor (Berk. } \\
\text { \& M.A. Curtis) R.H. Petersen }\end{array}$ & $16 / 7 / 12$ & $\begin{array}{l}\text { Saprobic on decaying wood log, } \\
\text { scattered, in group }\end{array}$ & Inedible \\
\hline DDUNPL231 & Coprinaceae & $\begin{array}{l}\text { Coprinus comatus (O.F. Müll.) } \\
\text { Pers. }\end{array}$ & $7 / 7 / 12$ & Saprobic on manure, in group & Inedible \\
\hline DDUNPL232 & Coprinaceae & $\begin{array}{l}\text { C. disseminates (Pers.:Fries) } \\
\text { J.E. Lange }\end{array}$ & $23 / 9 / 11$ & $\begin{array}{l}\text { Saprobic on rotting tree branch, in } \\
\text { group }\end{array}$ & Edible \\
\hline DDUNPL233 & Coprinaceae & C. domesticus (Bolton) Gray & $4 / 7 / 12$ & $\begin{array}{l}\text { Saprobic on decaying wood log, } \\
\text { gregariously in small troops }\end{array}$ & Inedible \\
\hline DDUNPL234 & Coprinaceae & C. lagopus (Fr.) Fr. & $7 / 7 / 12$ & $\begin{array}{l}\text { Saprobic on decaying wood log, } \\
\text { solitary to in group }\end{array}$ & Inedible \\
\hline DDUNPL235 & Coprinaceae & C. pellucidus P. Karst. & $19 / 9 / 11$ & $\begin{array}{l}\text { Saprobic on dead hardwood stump, } \\
\text { in group }\end{array}$ & Inedible \\
\hline DDUNPL236 & Coprinaceae & C. truncorum (Scop.) Fr. & $25 / 6 / 13$ & $\begin{array}{l}\text { Saprobic on decaying wood log, in } \\
\text { group }\end{array}$ & Inedible \\
\hline DDUNPL171 & Fomitopsidaceae & $\begin{array}{l}\text { Fomitopsis cajanderi } \\
\text { (P. Karst) Kotl. \& Pouzar }\end{array}$ & $6 / 9 / 13$ & Saprobic on dead wood, in group & Inedible \\
\hline DDUNPL172 & Fomitopsidaceae & F. pinicola (Sw.) P. Karst. & $5 / 8 / 12$ & $\begin{array}{l}\text { Saprobic on decaying tree } \\
(\text { Mangifera indica }) \text {, solitary to in } \\
\text { group }\end{array}$ & Inedible \\
\hline DDUNPL173 & Fomitopsidaceae & $\begin{array}{l}\text { Laetiporus sulphurous (Bull.) } \\
\text { Murrill }\end{array}$ & $26 / 9 / 11$ & $\begin{array}{l}\text { Saprobic on cut wood log forming } \\
\text { shelves }\end{array}$ & Edible \\
\hline DDUNPL174 & Fomitopsidaceae & Postia stiptica (Pers.) Jülich & $5 / 7 / 14$ & $\begin{array}{l}\text { Saprobic on decaying wood log, in } \\
\text { group }\end{array}$ & Inedible \\
\hline
\end{tabular}


Table 1 (continued)

\begin{tabular}{|c|c|c|c|c|c|}
\hline Voucher no. & Family & Macrofungi & $\begin{array}{c}\text { Date of } \\
\text { collection }\end{array}$ & Ecological habitat & Property \\
\hline DDUNPL189 & Ganodermataceae & $\begin{array}{l}\text { Ganoderma applanatum } \\
\text { (Pers.) Pat. }\end{array}$ & $23 / 9 / 11$ & $\begin{array}{l}\text { Parasitic on Tectona grandis, in } \\
\text { group }\end{array}$ & Inedible \\
\hline DDUNPL190 & Ganodermataceae & G. lucidum (Curtis) P. Karst. & $6 / 8 / 12$ & $\begin{array}{l}\text { Saprobic on dead woods, solitary or } \\
\text { in groups of } 2-3\end{array}$ & Edible \\
\hline DDUNPL191 & Ganodermataceae & G. tsugae Murrill & $21 / 10 / 11$ & Saprobic on decaying tree, solitary & Inedible \\
\hline DDUNPL271 & Geastraceae & Geastrum rufescens Pers. & $24 / 8 / 11$ & $\begin{array}{l}\text { Saprobic on soil among leaf litters, } \\
\text { solitary or in small groups of } 2-3\end{array}$ & Inedible \\
\hline DDUNPL162 & Helotiaceae & $\begin{array}{l}\text { Ascocoryne sarcoides (Jacq.) } \\
\text { J.W. Groves \& D.E. Wilson }\end{array}$ & $7 / 4 / 12$ & $\begin{array}{l}\text { Parasitic on Ficus racemosa, } \\
\text { gregarious, in group }\end{array}$ & Inedible \\
\hline DDUNPL196 & Hygrophoraceae & $\begin{array}{l}\text { Hygrophorus eburneus (Bull.) } \\
\text { Fr. }\end{array}$ & $23 / 9 / 11$ & $\begin{array}{l}\text { Saprobic on humus rich soil and on } \\
\text { straw heap, solitary }\end{array}$ & Edible \\
\hline DDUNPL192 & Hymenochaetaceae & $\begin{array}{l}\text { Coltricia cinnamomea (Jacq.) } \\
\text { Murrill }\end{array}$ & $18 / 10 / 11$ & $\begin{array}{l}\text { Saprobic on humid soil, growing } \\
\text { alone under hardwood }\end{array}$ & Inedible \\
\hline DDUNPL193 & Hymenochaetaceae & $\begin{array}{l}\text { Inonotus cuticularis (Bull.) P. } \\
\text { Karst. }\end{array}$ & $9 / 2 / 12$ & $\begin{array}{l}\text { Saprobic on decaying wood log } \\
\text { forming shelves, in group }\end{array}$ & Inedible \\
\hline DDUNPL194 & Hymenochaetaceae & I. hispidus (Bull.) P. Karst. & $6 / 7 / 14$ & $\begin{array}{l}\text { Saprobic to parasitic on trees, either } \\
\text { alone or forms shelves by joining } \\
\text { with other caps }\end{array}$ & Inedible \\
\hline DDUNPL195 & Hymenochaetaceae & I. radiatus (Sowerby) P. Karst. & $5 / 7 / 13$ & $\begin{array}{l}\text { Saprobic on decaying wood } \log \text {, in } \\
\text { group }\end{array}$ & Inedible \\
\hline DDUNPL257 & Inocybaceae & $\begin{array}{l}\text { Inocybe dulcamara (Pers.) P. } \\
\text { Kumm. }\end{array}$ & $4 / 7 / 14$ & $\begin{array}{l}\text { Saprobic on decaying leaf litter, } \\
\text { solitary to in group }\end{array}$ & Poisonous \\
\hline DDUNPL258 & Inocybaceae & I. fastigiata (Schaeff.) Quèl & $3 / 7 / 14$ & $\begin{array}{l}\text { Saprobic present on soil, solitary to } \\
\text { in group }\end{array}$ & Poisonous \\
\hline DDUNPL252 & Lentinaceae & Lentinus conatus Berk. & $4 / 7 / 14$ & $\begin{array}{l}\text { Saprobic on decaying wood log, in } \\
\text { group }\end{array}$ & Edible \\
\hline DDUNPL253 & Lentinaceae & L. squarrosulus Mont. & $23 / 6 / 14$ & $\begin{array}{l}\text { Saprobic on decaying wood log, in } \\
\text { group }\end{array}$ & Edible \\
\hline DDUNPL254 & Lentinaceae & L. tigrinus (Bull.) Fr. & $16 / 7 / 12$ & $\begin{array}{l}\text { Saprobic, growing on rotting wood } \\
\log \end{array}$ & Inedible \\
\hline DDUNPL268 & Lycoperdaceae & Bovista plumbea Pers. & $10 / 7 / 13$ & $\begin{array}{l}\text { Saprobic, scattered in troops in short } \\
\text { grasses }\end{array}$ & Edible \\
\hline DDUNPL269 & Lycoperdaceae & B. pusilla (Batsch) Pers. & $5 / 7 / 14$ & $\begin{array}{l}\text { Saprobic, scattered in troops in short } \\
\text { grasses }\end{array}$ & Inedible \\
\hline
\end{tabular}


Table 1 (continued)

\begin{tabular}{|c|c|c|c|c|c|}
\hline Voucher no. & Family & Macrofungi & $\begin{array}{c}\text { Date of } \\
\text { collection }\end{array}$ & Ecological habitat & Property \\
\hline DDUNPL270 & Lycoperdaceae & Lycoperdon perlatum Pers. & $7 / 7 / 12$ & $\begin{array}{l}\text { Saprobic, scattered along road side } \\
\text { in group }\end{array}$ & Edible \\
\hline DDUNPL259 & Lyophyllaceae & Calocybe gambosa (Fr.) Donk & $7 / 7 / 12$ & $\begin{array}{l}\text { Symbiotic, in association with } \\
\text { Azadirachta indica and Ficus } \\
\text { bengalensis, in group }\end{array}$ & Excellent edible \\
\hline DDUNPL260 & Lyophyllaceae & C.indica Purkay. \& A.Chandra & $18 / 6 / 13$ & $\begin{array}{l}\text { Saprobic on manure and husk } \\
\text { residue, in group }\end{array}$ & Excellent edible \\
\hline DDUNPL261 & Lyophyllaceae & $\begin{array}{l}\text { Termitomyces heimii } \mathrm{K} . \\
\text { Natarajan }\end{array}$ & $14 / 7 / 12$ & $\begin{array}{l}\text { Symbiotic in association with } \\
\text { termites nest }\end{array}$ & Excellent edible \\
\hline DDUNPL262 & Marasmiaceae & $\begin{array}{l}\text { Marasmius curreyi Berk. \& } \\
\text { Broome }\end{array}$ & $24 / 8 / 11$ & $\begin{array}{l}\text { Saprobic on rotting wood log, in } \\
\text { group }\end{array}$ & Inedible \\
\hline DDUNPL263 & Marasmiaceae & M. pulcherripes Peck & $5 / 7 / 14$ & Saprobic on litter, solitary to in group & Inedible \\
\hline DDUNPL264 & Marasmiaceae & M. sicci Murrill & $29 / 8 / 13$ & $\begin{array}{l}\text { Saprobic, on decaying wood } \log \text { and } \\
\text { leaf litter }\end{array}$ & Inedible \\
\hline DDUNPL175 & Meripilaceae & Grifola frondosa (Dicks.) Gray & $25 / 8 / 11$ & $\begin{array}{l}\text { Saprobic in association with } \\
\text { decaying Tectona grandis }\end{array}$ & Edible \\
\hline DDUNPL176 & Meruliaceae & $\begin{array}{l}\text { Abortiporus biennis (Bull.) } \\
\text { Singer }\end{array}$ & $24 / 6 / 13$ & $\begin{array}{l}\text { Parasitic with living trees especially } \\
\text { with Bambusa arundinacea, growing } \\
\text { alone or in group forming shelves }\end{array}$ & Inedible \\
\hline DDUNPL265 & Mycenaceae & $\begin{array}{l}\text { Favolaschia pustulosa } \\
\text { (Jungh.) Kuntze }\end{array}$ & $21 / 10 / 11$ & Saprobic on dead wood, gregarious & Inedible \\
\hline DDUNPL266 & Phallaceae & Mutinus caninus (Huds.) Fr. & $5 / 7 / 14$ & $\begin{array}{l}\text { Saprobic, growing } \\
\text { gregariously in garden }\end{array}$ & Inedible \\
\hline DDUNPL267 & Phallaceae & Phallus duplicates Bose & $6 / 7 / 13$ & $\begin{array}{llll}\text { Saprobic, } & \text { growing } & \text { alone } & \text { or } \\
\text { gregariously in garden } & & \end{array}$ & Inedible \\
\hline DDUNPL246 & Pleurotaceae & $\begin{array}{l}\text { Pleurotus cystidiosus O.K. } \\
\text { Mill. }\end{array}$ & $21 / 7 / 13$ & $\begin{array}{l}\text { Parasitic on Ficus benghalensis, in } \\
\text { group }\end{array}$ & Excellent edible \\
\hline DDUNPL247 & Pleurotaceae & P. dryinus (Pers.) P. Kumm. & $22 / 8 / 13$ & $\begin{array}{l}\text { Parasitic in association with } \\
\text { Dalbergia sissoo tree, in group }\end{array}$ & Inedible \\
\hline DDUNPL248 & Pleurotaceae & P. flabellatus Sacc. & $24 / 6 / 13$ & Saprobic on decaying wood, in group & Excellent edible \\
\hline DDUNPL249 & Pleurotaceae & P. florida (Mont.) Singer & $25 / 8 / 12$ & $\begin{array}{l}\text { Parasitic in association with Ficus } \\
\text { religiosa in group forming shelves }\end{array}$ & Edible \\
\hline DDUNPL250 & Pleurotaceae & P. ostreatus (Jacq.) P. Kumm. & $8 / 7 / 12$ & $\begin{array}{l}\text { Parasitic on Mangifera indica, } \\
\text { forming shelves }\end{array}$ & Excellent edible \\
\hline DDUNPL224 & Pluteaceae & Pluteus luteovirens Rea & $4 / 7 / 14$ & Saprobic on leaf litters, in group & Inedible \\
\hline DDUNPL225 & Pluteaceae & P. petasatus (Fries) Gillet & $19 / 8 / 11$ & Saprobic on wood debris, in group & Edible \\
\hline
\end{tabular}


Table 1 (continued)

\begin{tabular}{|c|c|c|c|c|c|}
\hline Voucher no. & Family & Macrofungi & $\begin{array}{c}\text { Date of } \\
\text { collection }\end{array}$ & Ecological habitat & Property \\
\hline DDUNPL226 & Pluteaceae & $\begin{array}{l}P \text {. rimulosus Kühner \& } \\
\text { Romagn. }\end{array}$ & $7 / 7 / 14$ & $\begin{array}{l}\text { Saprobic on rotting wood, solitary to } \\
\text { in small groups }\end{array}$ & Inedible \\
\hline DDUNPL227 & Pluteaceae & $\begin{array}{l}\text { Volvariella bombycina } \\
\text { (Schaeff.) Singer }\end{array}$ & $31 / 8 / 12$ & $\begin{array}{l}\text { Parasitic in association with Ficus } \\
\text { bengalensis tree, in group }\end{array}$ & Edible \\
\hline DDUNPL228 & Pluteaceae & $\begin{array}{l}\text { V. indica M.K. Saini, N.J. } \\
\text { Kaur \& N.S. Atri }\end{array}$ & $6 / 7 / 14$ & $\begin{array}{l}\text { Saprobic abundant in garden, in } \\
\text { group or scattered }\end{array}$ & Edible \\
\hline DDUNPL229 & Pluteaceae & $\begin{array}{l}\text { V. taylori (Berk. \& Broome) } \\
\text { Singer }\end{array}$ & $25 / 6 / 13$ & $\begin{array}{l}\text { Saprobic, scattered in open field, } \\
\text { solitary to in group }\end{array}$ & Edible \\
\hline DDUNPL230 & Pluteaceae & V. volvacea (Bull.) Singer & $3 / 9 / 12$ & $\begin{array}{l}\text { Saprobic on wheat straw or husk, in } \\
\text { group }\end{array}$ & Excellent edible \\
\hline DDUNPL177 & Polyporaceae & $\begin{array}{l}\text { Fomes hemitephrus } \\
\text { (Berk.) Cooke }\end{array}$ & $17 / 8 / 13$ & Parasitic on Tectona grandis, solitary & Inedible \\
\hline DDUNPL178 & Polyporaceae & $\begin{array}{l}\text { Funalia trogii (Berk.) } \\
\text { Bondartsev \& Singer }\end{array}$ & $5 / 7 / 14$ & $\begin{array}{lll}\text { Parasitic on } & \text { Artocarpus } \\
\text { heterophyllus, in group } & \end{array}$ & Inedible \\
\hline DDUNPL179 & Polyporaceae & Lenzites betulina (L.) Fr. & $4 / 4 / 12$ & $\begin{array}{l}\text { Saprobic on deadwood, in group with } \\
\text { overlapping cluster }\end{array}$ & Inedible \\
\hline DDUNPL180 & Polyporaceae & L. sepiaria (Wulfen) Fr. & $13 / 7 / 12$ & $\begin{array}{l}\text { Saprobic on decaying wood log, in } \\
\text { group }\end{array}$ & Inedible \\
\hline DDUNPL181 & Polyporaceae & $\begin{array}{l}\text { Microporus xanthopus (Fr.) } \\
\text { Kuntze. }\end{array}$ & $27 / 6 / 12$ & $\begin{array}{l}\text { Saprobic on deadwood of hard wood, } \\
\text { in group }\end{array}$ & Inedible \\
\hline DDUNPL182 & Polyporaceae & $\begin{array}{l}\text { Polyporus alveolaris } \\
\text { (DC.) Bondartsev \& Singer }\end{array}$ & $4 / 7 / 14$ & $\begin{array}{l}\text { Saprobic on decaying logs, solitary to } \\
\text { cluster }\end{array}$ & Inedible \\
\hline DDUNPL183 & Polyporaceae & P. brumalis (Pers.) Fr. & $27 / 3 / 12$ & Saprobic on decaying wood & Inedible \\
\hline DDUNPL184 & Polyporaceae & $\begin{array}{l}\text { Pycnoporus cinnabarinus } \\
\text { (Jacq.) P. Karst. }\end{array}$ & $27 / 3 / 12$ & $\begin{array}{l}\text { Saprobic on dead wood, solitary to } \\
\text { group }\end{array}$ & Inedible \\
\hline DDUNPL185 & Polyporaceae & Trametes elegans (Spreng.) Fr. & $6 / 8 / 13$ & $\begin{array}{l}\text { Saprobic on dead wood of hard } \\
\text { woods, solitary or in groups }\end{array}$ & Inedible \\
\hline DDUNPL186 & Polyporaceae & T. gibbosa (Pers.) Fr. & $5 / 12 / 11$ & $\begin{array}{l}\text { Saprobic on decaying wood log, } \\
\text { forming rosettes on top of cut stump }\end{array}$ & Inedible \\
\hline DDUNPL187 & Polyporaceae & T. hirsutus (Wulfen) Pat. & $5 / 7 / 14$ & Saprobic on dead woods, in group & Inedible \\
\hline DDUNPL188 & Polyporaceae & T. versicolor (L.) Lloyd & $13 / 09 / 12$ & $\begin{array}{l}\text { Saprobic on decaying wood log, } \\
\text { forming rosettes on top of cut stump }\end{array}$ & Inedible \\
\hline DDUNPL237 & Psathyrellaceae & $\begin{array}{l}\text { Coprinellus micaceus (Bull.) } \\
\text { Vilgalys, Hopple \& Jacq. } \\
\text { Johnson }\end{array}$ & $19 / 8 / 11$ & Saprobic on humus rich soil, in group & Edible \\
\hline
\end{tabular}


Table 1 (continued)

\begin{tabular}{|c|c|c|c|c|c|}
\hline Voucher no. & Family & Macrofungi & $\begin{array}{c}\text { Date of } \\
\text { collection }\end{array}$ & Ecological habitat & Property \\
\hline DDUNPL238 & Psathyrellaceae & $\begin{array}{l}\text { Coprinopsis atramentaria } \\
\text { (Bull.) Rehead, Vilgalys \& } \\
\text { Moncalvo }\end{array}$ & $26 / 9 / 11$ & $\begin{array}{l}\text { Saprobic, on rotting tree stump in } \\
\text { group }\end{array}$ & Edible \\
\hline DDUNPL239 & Psathyrellaceae & $\begin{array}{l}\text { C. cothurnata (Godey) } \\
\text { Redhead }\end{array}$ & $23 / 6 / 14$ & Coprophilous on animal dung & Inedible \\
\hline DDUNPL240 & Psathyrellaceae & $\begin{array}{l}\text { C. ephemeroides (D.C.) G. } \\
\text { Moreno }\end{array}$ & $4 / 7 / 14$ & $\begin{array}{l}\text { Saprobic on humus, solitary to in } \\
\text { group }\end{array}$ & Inedible \\
\hline DDUNPL241 & Psathyrellaceae & $\begin{array}{l}\text { C. foetidella (P.D. Orton) Atri, } \\
\text { A. Kaur \& M. Kaur }\end{array}$ & $23 / 6 / 14$ & Coprophilous on animal dung & Inedible \\
\hline DDUNPL242 & Psathyrellaceae & C. friesii (Quèlet) P. Karsten & 19/8/11 & $\begin{array}{l}\text { Saprobic on husk residue, solitary to } \\
\text { in group }\end{array}$ & Inedible \\
\hline DDUNPL243 & Psathyrellaceae & $\begin{array}{l}\text { Panaeolus ater (J.E. Lange) } \\
\text { Kühner \& Romagn. }\end{array}$ & $11 / 8 / 11$ & $\begin{array}{l}\text { Coprophilous on animal dung, in } \\
\text { small group }\end{array}$ & Inedible \\
\hline DDUNPL244 & Psathyrellaceae & $\begin{array}{l}\text { P. papilionaeous (Bull.) } \\
\text { Quèlel }\end{array}$ & $11 / 8 / 11$ & $\begin{array}{l}\text { Coprophilus on cow dung manure, in } \\
\text { group }\end{array}$ & Inedible \\
\hline DDUNPL245 & Psathyrellaceae & $\begin{array}{l}\text { Psathyrella automata (Fr.) } \\
\text { Quèl. }\end{array}$ & $11 / 8 / 11$ & $\begin{array}{l}\text { Saprobic on soil in grassland, } \\
\text { solitary, scattered }\end{array}$ & Inedible \\
\hline DDUNPL251 & Russulaceae & Russula sororia Fr. & $7 / 7 / 14$ & Saprobic on litter, solitary to in group & Edible \\
\hline DDUNPL165 & Schizophyllaceae & Schizophyllum commune Fries & $11 / 8 / 11$ & $\begin{array}{l}\text { Parasitic on Mangifera indica tree in } \\
\text { group }\end{array}$ & Inedible \\
\hline DDUNPL170 & Sparassidiaceae & Sparassis crispa (Wulf) Fr. & $18 / 8 / 11$ & $\begin{array}{l}\text { Saprobic on decaying wood log, in } \\
\text { group }\end{array}$ & Edible \\
\hline DDUNPL166 & Stereaceae & Stereum hirsutum (Wild.) Pers. & $9 / 5 / 13$ & Saprobic on deadwood, gregarious & Inedible \\
\hline DDUNPL197 & Tricholomataceae & $\begin{array}{l}\text { Clitocybe inversa (Scop.) } \\
\text { Quèl. }\end{array}$ & $18 / 8 / 11$ & $\begin{array}{l}\text { Saprobic on litter, solitary or in } \\
\text { groups }\end{array}$ & Inedible \\
\hline DDUNPL198 & Tricholomataceae & C. vibecina (Fr.) Quèl & $23 / 9 / 11$ & Saprobic on decaying litter, scattered & Poisonous \\
\hline DDUNPL199 & Tricholomataceae & $\begin{array}{l}\text { Collybia fuscopurpurea (Pers.) } \\
\text { P. Kumm. }\end{array}$ & $5 / 7 / 13$ & Saprobic on decaying litter, in group & Inedible \\
\hline DDUNPL200 & Tricholomataceae & Lepista flaccid (Sowerby) Pat. & $4 / 7 / 14$ & $\begin{array}{l}\text { Saprobic, abundant in mixed forest, } \\
\text { in group }\end{array}$ & Inedible \\
\hline DDUNPL201 & Tricholomataceae & L. luscina (Fr.) Singer & $31 / 8 / 12$ & Saprobic on humus, in group & Edible \\
\hline DDUNPL202 & Tricholomataceae & $\begin{array}{l}\text { Omphalina ericetorum (Pers.) } \\
\text { M. Lange }\end{array}$ & $7 / 7 / 14$ & $\begin{array}{l}\text { Saprobic on Bambusa arundinacea } \\
\text { leaf litter, in small groups }\end{array}$ & Inedible \\
\hline
\end{tabular}


Table 1 (continued)

\begin{tabular}{|c|c|c|c|c|c|}
\hline Voucher no. & Family & Macrofungi & $\begin{array}{c}\text { Date of } \\
\text { collection }\end{array}$ & Ecological habitat & Property \\
\hline DDUNPL203 & Tricholomataceae & O. postii (Fr.) Singer & $5 / 7 / 14$ & $\begin{array}{l}\text { Saprobic in grassy areas, solitary to } \\
\text { in group }\end{array}$ & Inedible \\
\hline DDUNPL161 & Tuberaceae & Tuber aestivum Vitt. & $10 / 7 / 13$ & $\begin{array}{l}\text { Saprobic under broad leaf trees on } \\
\text { calcareous soil }\end{array}$ & Excellent edible \\
\hline DDUNPL158 & Xylariaceae & $\begin{array}{l}\text { Daldinia concentric (Bolton) } \\
\text { Ces. \& De Not. }\end{array}$ & $29 / 8 / 11$ & $\begin{array}{l}\text { Saprobic on decaying wood log, in } \\
\text { group }\end{array}$ & Inedible \\
\hline DDUNPL159 & Xylariaceae & Xylaria hypoxylon (L.) Grev. & $3 / 7 / 14$ & Saprobic on rotting wood, scattered & Inedible \\
\hline DDUNPL160 & Xylariaceae & X. longiana $\mathrm{Rehm}$ & $27 / 6 / 13$ & Saprobic on decaying wood, in group & Inedible \\
\hline
\end{tabular}

Table 2 Shannon diversity index, Simpson diversity index, richness and evenness of macrofungi in Gorakhpur district (Tehsil wise)

\begin{tabular}{|c|c|c|c|c|c|c|}
\hline & Bansgaon & Campierganj & Gola & Khajni & Sadar & Sahjanwan \\
\hline No. of species (s) & 5 & 4 & 6 & 12 & 34 & 53 \\
\hline Total no. of individuals $(\mathrm{N})$ & 55 & 105 & 30 & 140 & 314 & 644 \\
\hline $\begin{array}{l}\text { Shannon diversity index } \\
(\mathrm{H})\end{array}$ & 1.13 & 0.16 & 1.43 & 2.08 & 3.04 & 3.61 \\
\hline $\begin{array}{l}\text { Simpson diversity index } \\
\text { (1-D) }\end{array}$ & 0.64 & 0.29 & 0.74 & 0.85 & 0.94 & 0.97 \\
\hline $\begin{array}{l}\text { Evenness } \\
\text { (E) }\end{array}$ & 0.82 & 0.49 & 0.62 & 0.87 & 0.86 & 0.90 \\
\hline
\end{tabular}


contains 12 species, Gola tehsil contains 6 species, Bansgaon tehsil contain 5 species and Campierganj tehsil contains 4 species (Fig. 6). Sahjanwan tehsil shows the maximum diversity index. The Shannon's diversity index and Simpson's diversity index were found to be 3.61 and 0.97 respectively and evenness to be 0.90 in Sahjanwan tehsil. The highest number of species in Sahjanwan tehsil was contributed by the frequent collections made during the study period compared to other study sites in present study. The difference of the occurrence of macrofungi in the various localities in the study area also can be attributed to several factors such as rainfall, quantities of suitable substrate, damp forests with constant high air humidity and type of forest. The lower number of macrofungi recorded in this study could be because; only one to two collecting visits were made to each site. In fact complete knowledge of the fungi for any locality would require continuous observation and collection over many years (Bolhassan et al. 2012). Species diversity and occurrence increase with the increasing number of visits over a longer period.

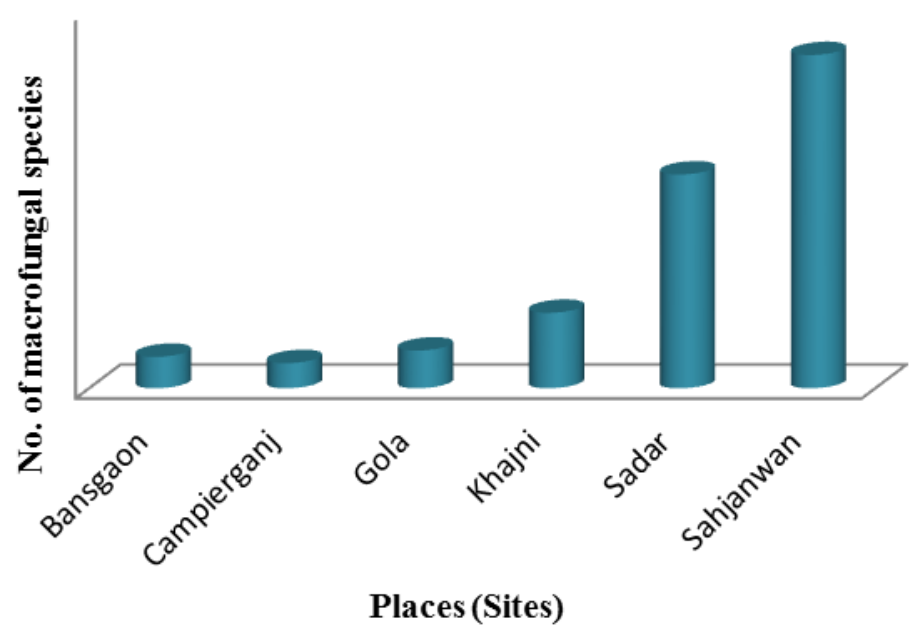

Fig. 6 - Number of macrofungi collected from different Tehsils of Gorakhpur district

\section{Conclusion}

North Eastern part of Uttar Pradesh with its varied topography, diverse vegetation and climatologic fluctuations can be successfully explored for the growth of macrofungi in wild habitat. In spite of the fact that this region is rich in resources of edible macrofungi, no planned effort has been made so far to collect and conserve them. There is vast scope of edible macrofungi which grow wild in forests and grasslands of this region. The moderate rainfall in this part supports a rich flora. These macrofungi can be widely used as food and medicine besides maintaining strength of ecosystem. Therefore it is very important to completely explore, document and conserve this natural wealth.

\section{Acknowledgements}

The authors wish to thank Head, Department of Botany DDU Gorakhpur University and Gorakhpur for providing necessary Lab. facilities.

\section{References}

Abolfazi P, Janardhana GR. 2012 - Diversity of Termitomyces in Kodagu and need for conservation. Journal of Advanced Laboratory Research in Biology 3, 54-57.

Ainsworth GC. 1971 - Ainsworth and Bisby's dictionary of Fungi. CMI, Kew, Surrey, England.

Alexopolous CJ, Mims CW, Blackwell M. 1996 - Introductory Mycology. John Wiley \& Sons, New York. 
Bolhassan MH, Abdullah N, Sabaratnam V, Tsutomu H, Abdullah S, Rashid NMN, Musa M Y. 2012 - Diversity and distribution of polyporales in Peninsular Malaysia. Sains Malaysiana 41(2), 155-161.

Chandrawati, Singh P, Kumar N, Tripathi NN. 2014 - Macrofungal wealth of Kusumhi Forest of Gorakhpur, U.P., India. American International Journal of Research in Formal, Applied and Natural Sciences 5(1), 71-75.

Ellis MB, Ellis JB. 1990 - Fungi without Gills (Hymenomycetes and Gasteromycetes), London: Chapman and Hall.

Ghosh RN, Pathak NC, Tewari I. 1967 - Studies on Indian Agaricales. Indian Phytopathology 20, 237-242.

Ghosh RN, Pathak NC. 1965 - The genus Macrolepiota in India. Indian Phytopathology 18, 360362.

Jordan M. 1995 - The Encyclopedia of fungi of Britain and Europe, John Taylor Book Venture Ltd., Newton Abbbot, Devon.

Kumar R, Tapwal A, Pandey S, Rishi R. 2013 - Fungal diversity associated with bamboo litter from Bambusetum of rain forest research institute, North East India. Biodiversitas 14, 79-88.

Kuo M. 2001 - Making spore prints. Retrieved from the MushroomExpert.com Website :http://www.bluewillopages.com/mushroomexpert/herbarium.html.

Moser M. 1983 - Keys to Agarics and Boleti. Stuttgart: Gustav Fischer Verlag.

Phillips R. 1981 - Mushrooms and other fungi of Great Britain \& Europe. London: Pan Books.

Priyamvada H, Akila M, Singh RK, Ravikrishna R, Verma RS, Philip L, Marathe RR, Sahu LK, Sudheer KP, Gunthe SS. 2017 - Terrestrial Macrofungal Diversity from the Tropical Dry Evergreen Biome of Southern India and Its Potential Role in Aerobiology. PLoS ONE 12(1): e0169333. doi:10.1371/journal.pone.0169333

Pushpa H, Purushothama KB. 2012 - Biodiversity of mushrooms in and around Bangalore (Karnataka), India. American-Eurasian Journal of Agricultural and Environmental Sciences 12(6), 750-759.

Sohi HS, Kumar S, Seth PK. 1964 - Some interesting fleshy fungi from Himachal Pradesh. Indian Phytopathology 17, 317-322.

Sohi HS, Kumar S, Seth PK. 1965 - Some interesting fleshy fungi from Himachal Pradesh. Journal of Indian Botanical Society 54, 69-73.

Tapwal A, Kumar R, Pandey S. 2013 - Diversity and frequency of macrofungi associated with wet ever green tropical forest in Assam, India. Biodiversitas 14, 73-78.

Vishwakarma P, Singh P, Mishra P, Tripathi NN. 2014 - Diversity of Some Wild Mushroom from Gorakhpur, Uttar Pradesh, India. Int. J. Pharm. Life Sci 5(7), 3643-3647.

Vishwakarma P, Singh P, Tripathi NN 2016 - Nutritional and antioxidant properties of wild edible macrofungi from North-Eastern Uttar Pradesh, India. Indian Journal of Traditional Knowledge 15(1), 143-148.

Vishwakarma P, Tripathi NN, Singh P 2017a - A checklist of macrofungi of Gorakhpur District, U.P. India. Current Research in Environmental \& Applied Mycology 7(2), 109-120.

Vishwakarma P, Singh P, Tripathi NN 2017b - In-vitro antioxidant activity and nutritional value of four wild oyster mushroom collected from North-Eastern part of Uttar Pradesh. Mycosphere 8(4), 592-602. 